\title{
TABU SEARCH HEURISTIC FOR FACILITY LAYOUT PROBLEM WITH ASILE STRUCTURE DESIGN
}

\author{
Pattanapong Praykaw a,*, Sakda Khamjan ${ }^{\text {b }}$, Supachai Pathumnakul a \\ ${ }^{a}$ Supply Chain and Logistics Systems Research Unit, Department of Industrial Engineering, Faculty of Engineering, \\ KhonKaen University, Khon Kaen, 40002,Thailand \\ ${ }^{\mathrm{b}}$ Faculty of Science and Engineer, Kasetsart University Chalermphrakiat Sakonnakhon Province Campus, \\ Sakhonnakhon 47000, Thailand \\ *Ppraykaw9@gmail.com
}

\begin{abstract}
Conventionally, distances between departments in facility layout problem (FLP) are estimated based on either Euclidean or Rectilinear methods. This assumption lessens the efficiency of layout. In this paper, the approach based on tabu search (TS) was developed to solve FLP by concurrently determining aisles for material handling between departments. The travelling distance between departments was estimated by shortest route on aisles. The objective function is to minimize the total travelling distance. The performance of the proposed TS is examined by comparing with the randomized solutions in a set of the example cases. The TS approach provides good solution with reasonable computation time even in the large size problem.
\end{abstract}

Keywords: aisle distance, facility layout problem, tabu search.

\section{Introduction}

One of the main objectives of the facility layout problem (FLP) is to locate the positions of departments that provide the minimum materials handling cost. ${ }^{(1)}$ It is widely known that the FLP is classified as the NP-hard problem and the heuristic methods are normally applied to solve the problems. ${ }^{(2)}$ There are several meta heuristics which have been employed to solve the problems such as Genetic Algorithms (GA), Simulated Annealing (SA) and Tabu Search (TS). ${ }^{(3)}$ Two of the most popular ones are $\mathrm{GA}^{(4,5,6,7)}$ and $\mathrm{TS}^{(1,3,8,9,10)}$. Moreover, most of the FLP research works in literature were assumed that the distances between departments were measured by either Euclidean or Rectilinear distances ${ }^{(11)}$ This measurement assumption often results in inaccurate distances in a plant layout design since, in practice, the material handling equipment travels through the aisle structure or an aisle network.

Based on the study of Marvin ${ }^{(12)}$ the performance of TS was better than the other metaheuristics such as GA and simulated annealing (SA) in solving all types of FLP.

In this paper, the approach based on TS is developed to solve FLP by concurrently determining aisles for material handling between departments. The travelling distances between departments are calculated by selecting the shortest route on aisles. The objective function is to minimize total travelling distance between departments.

\section{Problem description}

In order to efficiently apply the TS based heuristic, some characteristics of the problem are described and defined

\subsection{Objective function}

The objective function is to minimize total travelling distance as shown in equation (1)

$$
\text { Minimize } \sum_{i=0}^{M} \sum_{j=0}^{M} F_{i j} D_{i j}
$$

$F_{i j} \quad$ amount of material flow between departments $i$ and $j(i, j=1,2, \ldots, M)$.

$D_{i j} \quad$ travelling distance between departments $i$ and $j(i$ $, j=1,2, . ., M$ ).

$M \quad$ total number of departments required 


\subsection{Area and shape of departments}

All departments are assumed to be in rectangular shape which can be described by width (X) and depth (Y) of the department as shown in Fig. 1. The required area of a department equals to the product of $\mathrm{X}$ and $\mathrm{Y}$. In this paper, we allow $\mathrm{X}$ and $\mathrm{Y}$ to be variables by providing the minimum values of $\mathrm{X}$ and $\mathrm{Y}$. It means that the suitable $\mathrm{X}$ and $\mathrm{Y}$ should be at least equal to the specified minimum values of $\mathrm{X}$ and $\mathrm{Y}$,

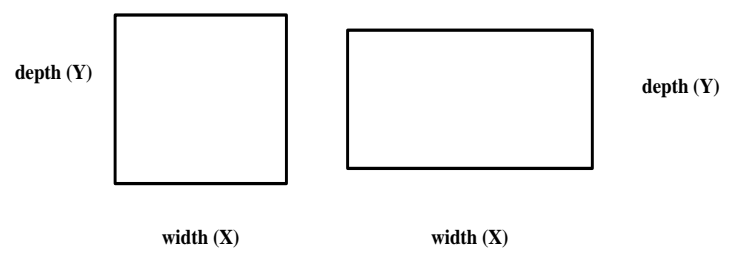

Fig. 1. The shapes of department represented by X and

\subsection{Row and column based layout}

A multi-row configuration for the layout is considered. The layout structure is described in terms of the numbers of rows and columns. For example, if a layout with three rows and three columns is required, then the layout consists of two horizontal aisles and two vertical aisles. Departments located on the same row have identical depths. The summation of the width of the departments in each low in the same column needs to be equal. The input/output points are located at the centroids of the departments as shown in Fig. 2

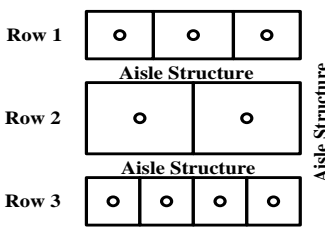

Column 1

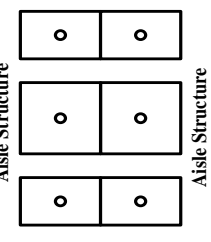

Column 2

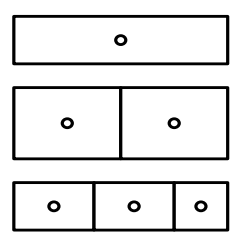

Column 3
Fig. 2. The layout based on 3 rows and 3 columns.

\subsection{Inter-department distance measurement}

Distance between departments is measured from the centroid of the departments and following the rules below which are used to incorporate the aisle distance.

(1) The distance between a pair of departments located on different rows and columns is rectilinear

(2) The distance between a pair of departments located contiguously on the same row is rectilinear

(3) The distance between a pair of departments located on the same row and are not contiguous to each other is the sum of the rectilinear distance and the vertical distance between the centroids of the departments and the centroids of the closest horizontal aisle

(4) The distance between a pair of departments on the same column but different aisles is the sum of vertical distance between the centroids of the departments and the horizontal distances between the centroids of the departments and the centroids of the vertical aisles that provide the shortest horizontal distance.

The distance measurement can be depicted as shown in Fig. 3

\section{Tabu Search (TS)}
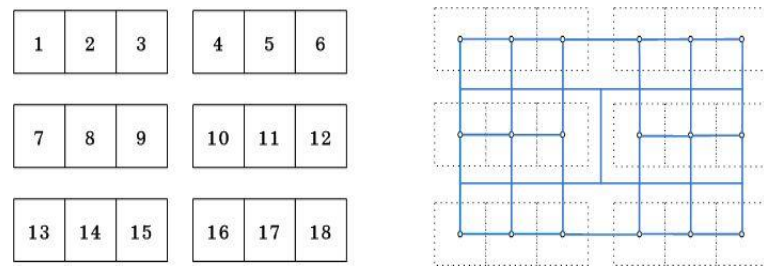

Fig. 3. Distance measurement between departments.

In this paper, the tabu search (TS) approach ${ }^{(5)}$ is employed to solve this specific FLP. The details of the tabu search approach applied to this problem are described as follows

Solution pattern: the solution of the problem is formed in $4 \times M$ matrix, where $M$ is the number of departments. The first row, I, is department number. The second row, $R$, is the row number while the third row, $\mathrm{C}$, is the column number. The last row, N, is the position number in the row. For example, the solution presented in Fig. 4(a), the department 4 is located at the second position of the row 1 and column 1 of the layout.

Neighborhood: the swap move operation is used to structure the neighborhood of the search. This move is the interchange of departments in solution pattern. The swapping operation will be described in the later example. For each iteration, all department pairs are swapped. The swap pair providing the best solution is selected to be the solution of the iteration and listed as tabu list.

Tabu list: a tabu list with a fixed iterations. The recent moves are stored in this list. The swap operation between two departments that have been recently swapped will be prohibited for the next $\mathrm{M}$ iterations.

Termination criterion: the tabu search is stopped when there is not any better solution than the current best solution for 5 consecutive iterations. The current best solution is the solution of the heuristic. 
The algorithm is to swap the positions of two departments to find the better solution. To avoid becoming trapped in local optima, the recently swapped pair of departments will be prohibited or stored in the tabu list with a fixed tenure of iterations. The algorithm will be repeated until the termination criterion is met. The algorithm could be described by the example with 6 departments, 2 rows and 1 column layout (see Table 1-4). Assume that the initial solution is randomly generated as shown in Table 1. Department 6 is located at the first position (i.e., $\mathrm{N}=1$ ) of the row 1 (i.e., $\mathrm{R}=1$ ) and column 1 (i.e., $\mathrm{C}=1$ ), while the department 4 is at the position 2 (i.e., $\mathrm{N}=2$ ) of the row 1 (i.e., $\mathrm{R}=1$ ) and column 1 (i.e., $\mathrm{C}=1$ ). The rests of departments could be described as the same manner. The layout of initial solution (Table 1) can be predicted as Fig. 4(a). In the first iteration, all department pairs (i.e., 6-4, 6-3, 6-5, 6-1, 6-2, 4$3,4-5,4-1,4-2,3-5,3-1,3-2,5-1,5-2,1-2$ ) are examined to be swapped. For example, in case where the departments 6 and 4 are to be swapped (see Table 2). The $\mathrm{R}$ and $\mathrm{C}$ of these two departments are randomly generated. Suppose the new $\mathrm{R}$ and $\mathrm{C}$ are as in Table 3, and then the position $\mathrm{N}$ are rearranged based on new $\mathrm{R}$ and $\mathrm{C}$ values. That is department 6 should be assigned in the first position of the row 1 column 1 while the department 4 is located at the second position of the row 2 column 1 (see Table 4). The layout is as shown in Fig. 4(b). The developed algorithm based on tabu search heuristic is more described in the flowchart (see Fig. 5)

Table 1. Initial solution.

\begin{tabular}{|c|c|c|c|c|c|c|}
\hline $\mathrm{I}$ & 6 & 4 & 3 & 5 & 1 & 2 \\
\hline $\mathrm{R}$ & 1 & 1 & 2 & 2 & 2 & 2 \\
\hline $\mathrm{C}$ & 1 & 1 & 1 & 1 & 1 & 1 \\
\hline $\mathrm{N}$ & 1 & 2 & 1 & 2 & 3 & 4 \\
\hline
\end{tabular}

Table 2. Departments 4 and 6 are swapped.

\begin{tabular}{|l|l|l|l|l|l|l|}
\hline$I$ & 4 & 6 & 3 & 5 & 1 & 2 \\
\hline$R$ & 1 & 1 & 2 & 2 & 2 & 2 \\
\hline$C$ & 1 & 1 & 1 & 1 & 1 & 1 \\
\hline$N$ & 1 & 2 & 1 & 2 & 3 & 4 \\
\hline
\end{tabular}

Table 3. Row and column numbers of departments 4 and 6 are randomly generated.

\begin{tabular}{|l|l|l|l|l|l|l|}
\hline$I$ & 4 & 6 & 3 & 5 & 1 & 2 \\
\hline$R$ & 2 & 1 & 2 & 2 & 2 & 2 \\
\hline$C$ & 1 & 1 & 1 & 1 & 1 & 1 \\
\hline$N$ & 1 & 2 & 1 & 2 & 3 & 4 \\
\hline
\end{tabular}

Table 4. Positions of departments $(\mathrm{N})$ are rearranged.

\begin{tabular}{|l|l|l|l|l|l|}
\hline$I$ & 6 & 3 & 4 & 5 & 1 \\
\hline$R$ & 1 & 2 & 2 & 2 & 2 \\
\hline$C$ & 1 & 1 & 1 & 1 & 1 \\
\hline$N$ & 1 & 1 & 2 & 3 & 4 \\
\hline & $\mathbf{3}$ & $\mathbf{5}$ & $\mathbf{1}$ & $\mathbf{2}$ \\
\hline & $\mathbf{6}$ & $\mathbf{4}$ & \\
\hline
\end{tabular}

(a) the layout following the Table 1 .

\begin{tabular}{|l|c|c|c|c|}
\hline $\mathbf{3}$ & $\mathbf{4}$ & $\mathbf{5}$ & $\mathbf{1}$ & $\mathbf{2}$ \\
\hline \multicolumn{4}{|c|}{ Aisle Structure } \\
\hline $\mathbf{6}$ & \multicolumn{3}{|c|}{} \\
\hline
\end{tabular}

(b) the layout following Table 4

Fig 4. The layout of the example case

\section{Result and discussion}

In this section, 14 problem cases were used to examine the performance and practicality of the proposed heuristic. The problem cases consisted of 6, 10, 15 departments. The number of specified rows and columns in the layout were varied between 2 to 3 rows and 1 to 3 columns, respectively. In each case, 5 problems were randomly generated. The performances of the proposed algorithm were compared to in the randomized solutions which were the best solution from a set of randomly generated solutions. The solutions from TS based approach were approximately $27 \%$ better than the randomized ones on average. The results are as shown in Table 5. The computation time was quite promising. The longest one was about 8 hours in the case of 15 departments with 3 rows and 3 columns, but most of the cases were less than 30 minute 


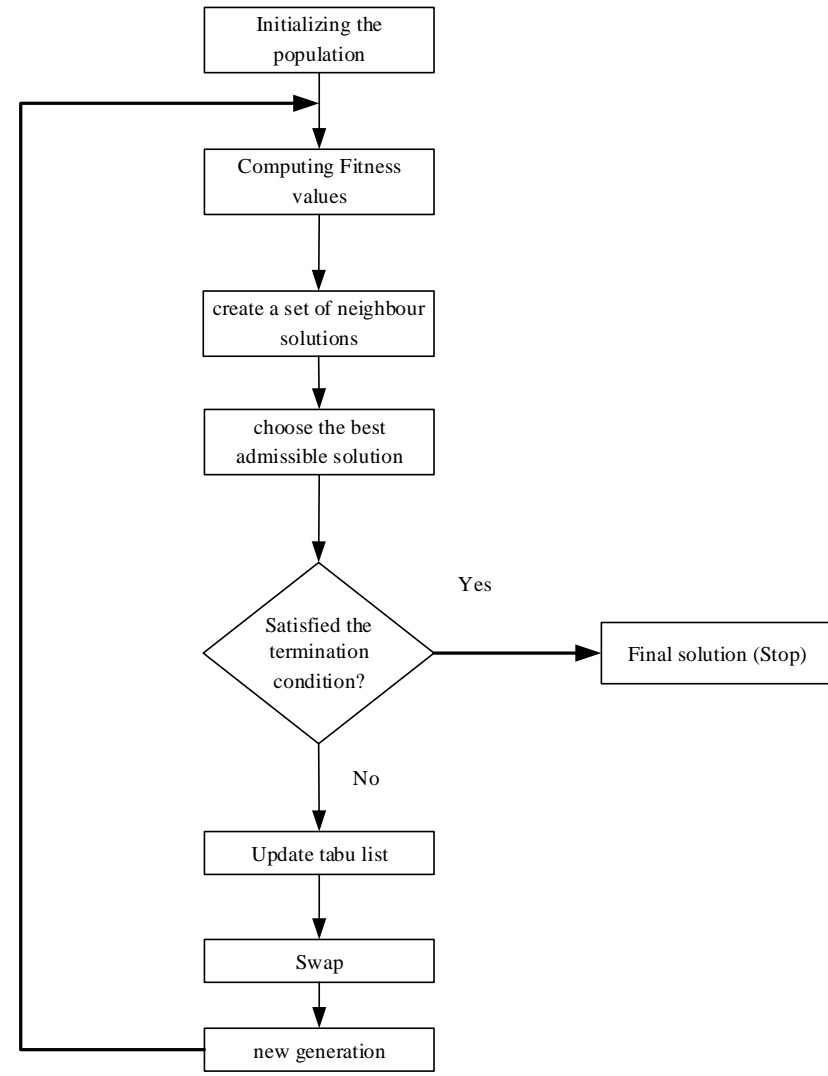

Fig. 5.The flowchart of the algorithm.

Table 5. The comparison results.

\begin{tabular}{|c|c|c|c|c|}
\hline $\begin{array}{c}\text { Problem } \\
\text { case }\end{array}$ & $\begin{array}{c}\text { No. of } \\
\text { departments }\end{array}$ & $\begin{array}{c}\text { No. of } \\
\text { Rows }\end{array}$ & $\begin{array}{c}\text { No. of } \\
\text { Columns }\end{array}$ & $\begin{array}{c}\text { Performance of } \\
\text { TS better than } \\
\text { randomized } \\
\text { method on } \\
\text { average (\%) }\end{array}$ \\
\hline 1 & 6 & 2 & 1 & 23 \\
\hline 2 & 6 & 2 & 2 & 21 \\
\hline 3 & 6 & 2 & 3 & 14 \\
\hline 4 & 6 & 3 & 1 & 33 \\
\hline 5 & 6 & 3 & 2 & 37 \\
\hline 6 & 10 & 2 & 1 & 28 \\
\hline 7 & 10 & 2 & 2 & 13 \\
\hline 8 & 10 & 2 & 3 & 21 \\
\hline 9 & 10 & 3 & 1 & 66 \\
\hline 10 & 10 & 3 & 2 & 48 \\
\hline 11 & 10 & 3 & 3 & 40 \\
\hline 12 & 15 & 2 & 3 & 16 \\
\hline 13 & 15 & 3 & 1 & 12 \\
\hline 14 & 15 & 3 & 2 & 13 \\
\hline
\end{tabular}

\section{Conclusions}

In this paper, the algorithm based on tabu search heuristic was applied to the facility layout problem with aisle structure design. The layout of departments and aisles were concurrently designed based on the specified number of rows and columns. The objective was to minimize the total real travelling distance. The performance of the model was compared to the randomized layout. The results showed that the proposed heuristic was efficient and practical in solving this specific FLP. Even though the computation time of some large size problems was quite long, but, in most of cases, the layouts could be obtained within reasonable computation time.

To increase the efficiency of solving this type of FLP, the other metaheuristics such as GA, SA and the particle swarm optimization (PSO) should be explored in future research

\section{References}

(1) N. Bozorgi, M. Abedzadeh, and M. Zeinali : “Tabu search heuristic for efficiency of dynamic facility layout problem” Int J Adv Manuf Technol, Vol. 77, pp. 689703,2015

(2) Min Zhang, Selçuk Savas, Rajan Batta, and Rakesh Nagi : "Facility placement with sub-aisle design in an existing layout", European Journal of Operational Research, Vol. 197, pp.154-165, 2009

(3) Srisatja Vitayasak, and Pupong Pongcharoen : "Machine selection rules for designing multi-row rotatable machine layout considering rectangular-tosquare ratio", Journal of Applied Operational Research, Vol. 5, No.2, pp. 48-55, 2013

(4) Chan FTS, Chung SH, and Chan PLY : "Application of Genetic Algorithms with dominant genes in a distributed scheduling problem in flexible manufacturing systems", Int J Prod Res, Vol. 44, NO.3, pp. 523-543, 2006

(5) Giuseppe Aiello, Giada La Scalia, and Mario Enea : "A multi objective genetic algorithm for the facility layout problem based upon slicing structure encoding", Expert Systems with Applications, Vol. 39, pp.1035210358,2012 
(6) S. Jannat, A.A. Khaled, and Sanjoy Kumar Paul : "Optimal Solution for Multi-Objective Facility Layout Problem Using Genetic Algorithm”, International Conference on Industrial Engineering and Operations Management, 2010

(7) Xiaodan Wu a, Chao-Hsien Chu b, Yunfeng Wang, and Weili Yan : "A genetic algorithm for cellular manufacturing design and layout", European Journal of Operational Research, Vol. 181, pp.156-167, 2007

(8) Hamed Samarghandi, and Kourosh Eshghi : "An efficient tabu algorithm for the single row facility layout problem", European Journal of Operational Research, Vol. 205 , pp.98-105, 2010

(9) Lou Y. Liang, and Wen C. Chao : "The strategies of tabu search technique for facility layout optimization", Automation in Construction, Vol. 17 , pp.657-66, 2008

(10) Nearchou, A. C : "Meta-heuristics from nature for the loop layout design problem", International Journal of Production Economics, Vol. 101, pp. 312-328, 2006

(11)Zhang M, Savas S, Batta R, Nagi R: "Facility placement with sub-aisle design in an existing layout", European Journal of Operational Research, Vol. 197, No. 2, pp.154-165, 2009

(12) Marvin A. Arostegui Jr., Sukran N. Kadipasaoglu, Basheer M. Khumawala : "An empirical comparison of Tabu Search, Simulated Annealing, and Genetic Algorithms for facilities location problems", Original Research Article International Journal of Production Economics, Vol. 103, No.2, pp 742-754, 2006 